hep-th/9905211

HUTP-99/A023

\title{
Holography for Coset Spaces
}

\author{
Ruth Britto-Pacumio, 1 Andrew Strominger 2 and Anastasia Volovich ${ }^{3} \dagger$ \\ Department of Physics \\ Harvard University \\ Cambridge, MA 02138
}

\begin{abstract}
$\mathrm{M} /$ string theory on noncompact, negatively curved, cosets which generalize $A d S_{D+1}=$ $S O(D, 2) / S O(D, 1)$ is considered. Holographic descriptions in terms of a conformal field theory on the boundary of the spacetime are proposed. Examples include $S U(2,1) / U(2)$, which is a Euclidean signature $(4,0)$ space with no supersymmetry, and $S O(2,2) / S O(2)$ and $S O(3,2) / S O(3)$, which are Lorentzian signature $(4,1)$ and $(6,1)$ spaces with eight supersymmetries. Qualitatively new features arise due to the degenerate nature of the conformal boundary metric.
\end{abstract}

\footnotetext{
1 britto@boltzmann.harvard.edu

2 andy@planck.harvard.edu

3 nastya@physics.harvard.edu

$\dagger$ On leave from L. D. Landau Institute for Theoretical Physics, Kosigina 2, Moscow, Russia
} 


\section{Introduction}

In Maldacena's $A d S / C F T$ duality [1], M/string theory on $A d S$ is equivalent to a field theory on the boundary of $A d S$. This is a concrete example of the plausibly much more general holographic principle [2-4. The holographic description of $A d S$ gravity relies on very special properties of $A d S$, such as the fact that the ratio of the volume and surface area approaches a constant at large radius. Hence it is far from obvious how the holographic principle can be concretely realized in a general setting. Discussions of holography in cosmology have appeared in [5], in flat space in [6], and in negatively curved spaces other than $A d S$ in [7]10].

In this paper we propose a holographic description of $\mathrm{M} /$ string theory in a family of negatively curved symmetric spacetimes. $A d S_{D+1}$ can be represented as the

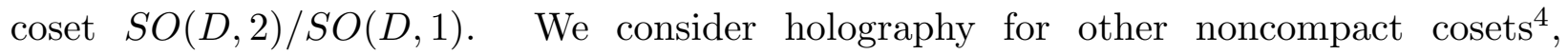
mainly $S U(2,1) / U(2)$, which is a signature $(4,0)$ space with no supersymmetry, and $S O(2,2) / S O(2)$ and $S O(3,2) / S O(3)$, which are signature $(4,1)$ and $(6,1)$ spaces with eight supersymmetries. There are many other similar noncompact cosets. These spaces have unusual features such as closed timelike curves but nevertheless provide an interesting and challenging arena in which to expand our understanding of holography.

An important new feature is that the conformal boundary metric for these cosets has zero eigenvalues. This feature also appears in the conformal boundary metric at null infinity in Minkowski space, and so may be pertinent in more physically interesting spacetimes. Despite the degeneracy of the conformal boundary metric there is a nondegenerate conformal boundary measure. We argue that this is enough to enable us to define the boundary theory via its correlators. We find that, as in $A d S$, the bulk isometries become conformal isometries of the boundary, and the boundary scalar operators and scalar correlation functions transform accordingly. (Similar results may hold for higher spin, but they are not explicitly investigated here.) Another generic feature, associated with the degeneracy of the boundary metric, is the appearance of an infinite-dimensional enlarged conformal symmetry group, in some ways analogous to the enlargement of $s o(2,2)$ to two copies of the Virasoro algebra on the boundary of $A d S_{3}$.

This paper is organized as follows. In section 2 we consider M-theory on $S U(2,1) / U(2) \times$ $S^{7}$. The geometry and symmetries of $S U(2,1) / U(2)$ and its conformal boundary are described. The boundary measure is conformally the standard round measure on $S^{3}$, while

4 Holography for the cases of vacua of the form $A d S \times X$, where $X$ is a compact coset space, has been studied in [11]. 
the conformal boundary metric has signature $(+, 0,0)$. The Einstein-Kähler deformations are discussed, following [12,13]. The compactification is shown to be free of tachyonic instabilities. A prescription is given, generalizing [1, 14, 15], for defining the correlators of the boundary conformal field theory as appropriately rescaled limits of bulk correlators. They are seen to be finite despite the degeneracy of the boundary metric. Two-point functions are explicitly computed using the $S U(2,1)$ conformal isometry group. Section 3 concerns IIB string theory on $S O(2,2) / S O(2) \times S^{5}$ and also briefly M-theory on $S O(3,2) / S O(3) \times S^{4}$.

These are both supersymmetric Lorentzian signature spacetimes. In the former case we propose that the appropriate boundary theory is a two-dimensional conformal field theory. In the final section 4 we conjecture a dual description in terms of conformal field theories from branes on spacetimes with degenerate metrics. We also describe how solitons can spontaneously break $S O(D, 2)$ conformal invariance down to a smaller subgroup, and suggest that at the duals to such configurations may in some cases be interesting Lorentzian cosets.

\section{M-theory on $S U(2,1) / U(2) \times S^{7}$}

In this section we consider M-theory compactified on $S U(2,1) / U(2) \times S^{7}$ and its holographic representation on the boundary of $S U(2,1) / U(2)$. This is a Euclidean space with no supersymmetry, as can be easily seen from the absence of a candidate supergroup. In subsection 2.1 we describe the bulk geometry of this space as well as the degenerate conformal geometry of the boundary. Relevant results relating the metric deformations to boundary data [12,13] are recalled in subsection 2.2. In 2.3 the mass of a scalar field is related to the quadratic Casimir of $S U(2,1)$ and it is shown that there are no tachyonic instabilities. In subsection 2.4 a modification of the $A d S / C F T$ prescription is given for constructing the scalar correlators of the conformal field theory on the boundary as limits of bulk correlators.

\subsection{Geometry of $S U(2,1) / U(2)$ and its conformal boundary}

The coset space $H=S U(2,1) / U(2)$ is topologically the open ball in $\mathbf{C}^{2}$ with the Bergman metric

$$
d s^{2}=\frac{d z_{1} d \bar{z}_{1}+d z_{2} d \bar{z}_{2}}{1-z_{1} \bar{z}_{1}-z_{2} \bar{z}_{2}}+\frac{1}{\left(1-z_{1} \bar{z}_{1}-z_{2} \bar{z}_{2}\right)^{2}}\left(\bar{z}_{1} d z_{1}+\bar{z}_{2} d z_{2}\right)\left(z_{1} d \bar{z}_{1}+z_{2} d \bar{z}_{2}\right),
$$


where $z_{1} \bar{z}_{1}+z_{2} \bar{z}_{2}<1$. This is a Kähler metric with Kähler potential

$$
K=-\frac{1}{2} \ln \left(1-z_{1} \bar{z}_{1}-z_{2} \bar{z}_{2}\right)
$$

Under the change of coordinates

$$
z_{1}=r \cos \frac{\theta}{2} e^{i(\varphi+\psi) / 2}, \quad z_{2}=r \sin \frac{\theta}{2} e^{-i(\varphi-\psi) / 2}
$$

this metric takes the form

$$
d s^{2}=\frac{d r^{2}}{\left(1-r^{2}\right)^{2}}+\frac{r^{2}}{4\left(1-r^{2}\right)}\left(\sigma_{1}^{2}+\sigma_{2}^{2}\right)+\frac{r^{2}}{4\left(1-r^{2}\right)^{2}} \sigma_{3}^{2},
$$

where the left-invariant one-forms are

$$
\begin{aligned}
\sigma_{1} & =\cos \psi d \theta+\sin \psi \sin \theta d \varphi \\
\sigma_{2} & =-\sin \psi d \theta+\cos \psi \sin \theta d \varphi \\
\sigma_{3} & =d \psi+\cos \theta d \varphi
\end{aligned}
$$

In this metric $r \in[0,1), \theta \in[0, \pi), \varphi \in[0,2 \pi)$, and $\psi \in[0,4 \pi)$. Defining $r=\tanh y$ yields yet another form of the metric,

$$
d s^{2}=d y^{2}+\frac{1}{4} \sinh ^{2} y\left(\sigma_{1}^{2}+\sigma_{2}^{2}\right)+\frac{1}{4} \sinh ^{2} y \cosh ^{2} y \sigma_{3}^{2} .
$$

The geometry (2.1) has an $S U(2,1)$ isometry group because the left action on the $S U(2,1)$ group manifold remains unbroken in the quotient by the right action of $U(2)$. This group is generated by the following eight Killing vectors.

$$
\begin{array}{cl}
H_{1}=z_{1} \partial_{z_{1}}-\bar{z}_{1} \partial_{\bar{z}_{1}}, & H_{2}=z_{2} \partial_{z_{2}}-\bar{z}_{2} \partial_{\bar{z}_{2}}, \\
L_{1}=z_{2} \partial_{z_{1}}-\bar{z}_{1} \partial_{\bar{z}_{2}}, & \bar{L}_{1}=\bar{z}_{2} \partial_{\bar{z}_{1}}-z_{1} \partial_{z_{2}}, \\
L_{2}=\partial_{z_{1}}-\bar{z}_{1} \bar{z}_{2} \partial_{\bar{z}_{2}}-\bar{z}_{1}^{2} \partial_{\bar{z}_{1}}, & \bar{L}_{2}=\partial_{\bar{z}_{1}}-z_{1} z_{2} \partial_{z_{2}}-z_{1}^{2} \partial_{z_{1}}, \\
L_{3}=\partial_{\bar{z}_{2}}-z_{1} z_{2} \partial_{z_{1}}-z_{2}^{2} \partial_{z_{2}}, & \bar{L}_{3}=\partial_{z_{2}}-\bar{z}_{1} \bar{z}_{2} \partial_{\bar{z}_{1}}-\bar{z}_{2}^{2} \partial_{\bar{z}_{2}} .
\end{array}
$$

The commutation relations between these generators are given in appendix A. So far the structure of $S U(2,1) / U(2)$ is qualitatively similar to Euclidean $A d S_{4}$, which is the coset $S O(4,1) / S O(4)$. However, the structure of the conformal boundary is quite different. The conformal boundary metric is determined (up to conformal transformations) by rescaling 
(2.6) by a singular function of $y$ such that the induced metric at the boundary $y=\infty$ is finite. Rescaling (2.6) by $64 e^{-4 y}$ yields the induced metric on a surface of constant $y$,

$$
d s^{2}=4 e^{-2 y}\left(1-e^{-2 y}\right)^{2}\left(\sigma_{1}^{2}+\sigma_{2}^{2}\right)+\left(1-e^{-4 y}\right)^{2} \sigma_{3}^{2} .
$$

This is a squashed three-sphere. As the boundary is approached, the squashing becomes more and more severe, until finally at the boundary it degenerates to

$$
d s^{2}=\sigma_{3}^{2}=(d \psi+\cos \theta d \varphi)^{2} .
$$

This metric has signature $(+, 0,0)$.

Degenerate conformal metrics have appeared in other contexts. For example, the boundary of $A d S_{4} \times S^{7}$ is $S^{3} \times S^{7}$, but after conformal rescaling, the metric on the $S^{7}$ factor is degenerate, and one has an effectively three-dimensional metric. An analogous interpretation of (2.12) as a metric on a one-dimensional space does not seem possible, since the one-form $\sigma_{3}$ is not closed. Another example is the conformal metric at null infinity of Minkowski space, which has signature $(0,+,+)$. This last example suggests that the problem of degenerate boundary metrics may be relevant for flat space holography.

Since the metric (2.12) is degenerate, the associated measure on the boundary vanishes. It is nevertheless possible to define a conformal measure on the boundary. Rescaling (2.6) by $2^{14 / 3} e^{-8 y / 3}$, one finds the finite induced volume form at the boundary

$$
\epsilon_{3}=\sigma_{1} \wedge \sigma_{2} \wedge \sigma_{3}
$$

and associated measure

$$
d^{3} \Omega=\sin \theta d \theta d \psi d \phi
$$

Global scale transformations in the boundary theory are induced by shifts of $y$. Since different powers of $e^{y}$ are required to make the induced measure and metric finite, their scaling dimensions will not be related by the usual factor of $2 / 3$ (in three dimensions). Rather the scale transformations are

$$
\begin{aligned}
d s_{3}^{2} & \rightarrow \Omega^{2}(\hat{x}) d s_{3}^{2}, \\
\epsilon_{3} & \rightarrow \Omega^{2}(\hat{x}) \epsilon_{3},
\end{aligned}
$$

where $\hat{x}$ is a coordinate on the $S^{3}$ boundary. 
Despite the degeneracy of the metric, the conformal Killing equation

$$
\mathcal{L}_{\xi} g_{a b}=f(\hat{x}) g_{a b},
$$

which does not involve the inverse metric, is well-defined. Conformal Killing vectors on the boundary with an $s u(2,1)$ Lie bracket algebra are obtained by restrictions of (2.7)-(2.10), namely

$$
\begin{gathered}
h_{1}=-i\left(\partial_{\varphi}+\partial_{\psi}\right), \quad h_{2}=i\left(\partial_{\varphi}-\partial_{\psi}\right), \\
l_{1}=-e^{-i \varphi}\left(\partial_{\theta}+\frac{i}{\sin \theta}\left(\partial_{\psi}-\cos \theta \partial_{\varphi}\right)\right), \quad \bar{l}_{1}=-e^{i \varphi}\left(\partial_{\theta}-\frac{i}{\sin \theta}\left(\partial_{\psi}-\cos \theta \partial_{\varphi}\right)\right), \\
l_{2}=-e^{-i(\varphi+\psi) / 2}\left(\sin \frac{\theta}{2} \partial_{\theta}+\frac{i}{2 \cos \frac{\theta}{2}}\left(\partial_{\varphi}+\left(1+2 \cos ^{2} \frac{\theta}{2}\right) \partial_{\psi}\right)\right), \\
\bar{l}_{2}=-e^{i(\varphi+\psi) / 2}\left(\sin \frac{\theta}{2} \partial_{\theta}-\frac{i}{2 \cos \frac{\theta}{2}}\left(\partial_{\varphi}+\left(1+2 \cos ^{2} \frac{\theta}{2}\right) \partial_{\psi}\right)\right), \\
l_{3}=e^{-i(\varphi-\psi) / 2}\left(\cos \frac{\theta}{2} \partial_{\theta}-\frac{i}{2 \sin \frac{\theta}{2}}\left(\partial_{\varphi}-\left(1+2 \sin ^{2} \frac{\theta}{2}\right) \partial_{\psi}\right)\right) . \\
\bar{l}_{3}=e^{i(\varphi-\psi) / 2}\left(\cos \frac{\theta}{2} \partial_{\theta}+\frac{i}{2 \sin \frac{\theta}{2}}\left(\partial_{\varphi}-\left(1+2 \sin ^{2} \frac{\theta}{2}\right) \partial_{\psi}\right)\right)
\end{gathered}
$$

One may check explicitly that the function $f$ in (2.16) is

$$
f(\hat{x})=\nabla_{m} \xi^{m}
$$

$f$ vanishes for $(2.17)-(2.18)$ which are the $S U(2) \times U(1)$ isometries of the boundary. We note that despite the degeneracy of the metric the covariant divergence is still well-defined. When the metric is nondegenerate, one can easily show that the coefficient on the right hand side of (2.16) is always $\frac{2}{D}$ (in $D$ dimensions) with $f$ defined in (2.23), simply by contraction with the inverse metric. However, the metric (2.12) does not have an inverse so no such demonstration is possible, and we remarkably find the same function with a different coefficient. These conformal Killing vectors also preserve the measure

$$
\mathcal{L}_{\xi} \epsilon_{3}=f(\hat{x}) \epsilon_{3}
$$

Here we encounter the standard conformal transformation law for a nondegenerate measure.

Due to the degeneracy of the metric, there are infinitely many conformal Killing vectors in addition to (2.17)-(2.22), as detailed in appendix B. Because they do not arise from 
the isometries of the bulk, there is no reason to expect that they annihilate the vacuum or provide simple relations among the correlators of the boundary theory. A somewhat similar situation occurs in $A d S_{3}$, for which the bulk isometries are $S L(2, R) \times S L(2, R)$, but the conformal Killing vectors of the boundary theory generate two copies of the Virasoro algebra. In that case the existence of the infinite-dimensional Virasoro algebra of course has profound consequences for the boundary theory. We do not know if that is also the case for $S U(2,1) / U(2)$.

\subsection{Deformations}

In this section we discuss deformations of the metric $S U(2,1) / U(2)$ which preserve the Einstein equations but in general destroy the isometries. There is a well-developed theory of such deformations. A key relevant result [12,13] is that for any strictly pseudoconvex domain $\Omega$ in $\mathbf{C}^{n}$ with a smooth boundary $\partial \Omega$ there is a unique complete Einstein-Kähler metric. The Kähler potential is 5

$$
K=-\frac{1}{2} \ln s,
$$

where $s$ is a solution of Fefferman's equation (slightly rewritten)

$$
(-s)^{n+1} \operatorname{det} \partial_{i} \partial_{\bar{j}} \ln s=-1
$$

subject to the Dirichlet boundary condition

$$
\left.s\right|_{\partial \Omega}=0 .
$$

This boundary condition ensures that the boundary points are an infinite distance from the interior.

The case of $S U(2,1) / U(2)$ arises from the domain in $\mathbf{C}^{2}$ bounded by the $S^{3}$ given by

$$
v \equiv 1-\left|z_{1}\right|^{2}-\left|z_{2}\right|^{2}=0
$$

The solution of $(2.26)$ is then simply

$$
s=v .
$$

It is easy to check that the resulting Kähler metric is indeed the Bergman metric (2.1).

A Kähler-Einstein deformation of the Bergman metric can then be succinctly described by deforming the equation for the boundary (2.28), for example by a polynomial in $(z, \bar{z})$. One can then find $s$ near the boundary in a power series expansion in $v$ with a $\ln v$ term.

5 The factor of $\frac{1}{2}$, not present in [13], is inserted to conform to the conventions of this paper. 


\subsection{Scalar fields and stability}

Consider a scalar field $\phi$ with mass $m$. The wave equation is

$$
\nabla^{2} \phi-m^{2} \phi=0
$$

where $\nabla^{2}$ is the Laplacian for the metric (2.4),

$$
\begin{gathered}
\nabla^{2}=\left(1-r^{2}\right)^{2} \partial_{r r}+\frac{\left(1-r^{2}\right)\left(3-r^{2}\right)}{r} \partial_{r}+ \\
\frac{4\left(1-r^{2}\right)}{r^{2}}\left(\partial_{\theta \theta}+\cot \theta \partial_{\theta}+\csc ^{2} \theta\left(\partial_{\varphi \varphi}-2 \cos \theta \partial_{\varphi \psi}+\left(1-r^{2} \sin ^{2} \theta\right) \partial_{\psi \psi}\right) .\right.
\end{gathered}
$$

The quadratic Casimir for $S U(2,1)$ is

$$
C_{I I}=-\frac{1}{2}\left\{L_{1}, \bar{L}_{1}\right\}+\frac{1}{2}\left\{L_{2}, \bar{L}_{2}\right\}+\frac{1}{2}\left\{L_{3}, \bar{L}_{3}\right\}+T^{2}+\frac{3}{4} Y^{2},
$$

where we redefined $Y=-H_{1}-H_{2}, T=\left(H_{2}-H_{1}\right) / 2$. Using the vector fields (2.7)-(2.10), we find that the Laplacian is proportional to the Casimir with a factor of 4 . Therefore the solutions of the wave equation for a scalar field of mass $m$ form a representation of $S U(2,1)$ with quadratic Casimir

$$
C_{I I}=\frac{m^{2}}{4}
$$

Next we use the $s u(2,1)$ algebra to classify the solutions of this equation. The representations were studied in [16]. The rank of $S U(2,1)$ is two and highest-weight representations are labelled by $(t, y)$, such that

$$
Y|\psi\rangle=y|\psi\rangle, \quad T|\psi\rangle=t|\psi\rangle
$$

Using the commutation relations $\left[L_{1}, \bar{L}_{1}\right]=-2 T, \quad\left[L_{2}, \bar{L}_{2}\right]=-\frac{3}{2} Y+T, \quad\left[L_{3}, \bar{L}_{3}\right]=\frac{3}{2} Y-T$, and the highest-weight conditions

$$
L_{1}|\psi\rangle=L_{2}|\psi\rangle=L_{3}|\psi\rangle=0
$$

we obtain from (2.32) the equation

$$
C_{I I}|\psi\rangle=t^{2}+2 t+\frac{3}{4} y^{2}|\psi\rangle
$$

or in terms of integers $(p, q)$ such that $t=\frac{1}{2}(p+q)$ and $y=\frac{1}{3}(p-q)$,

$$
m^{2}=\frac{4}{3}\left(p^{2}+q^{2}+p q\right)+4(p+q) .
$$


For the scalar field $\phi$, the highest-weight conditions imply $y=0$, or $p=q$ and we obtain the relation between the mass $m$ of the scalar field and the highest weight $p$ of the form

$$
m^{2}=4 p(p+2)
$$

The functional integral in the quantum theory includes all normalizable modes of $\phi$, even if they do not solve the wave equation. These can be characterized as eigenmodes $\phi_{k}$ of the Laplacian with eigenvalues $\lambda_{k}$ that obey

$$
-\nabla^{2} \phi_{k}+m^{2} \phi_{k}=\lambda_{k} \phi_{k}
$$

If there is a negative eigenvalue $\lambda_{k}$ with normalizable eigenmode $\phi_{k}$, fluctuations of $\phi_{k}$ are unstable. We wish to show that no such instabilities arise for M-theory on $S U(2,1) / U(2) \times$ $S^{7}$. Supersymmetry cannot be invoked since there are no appropriate covariantly constant spinors in this geometry.

At large $y$, the angular part of the Laplacian is exponentially suppressed, and $\phi_{k}$ obeys

$$
\frac{1}{\sqrt{g}} \partial_{y} \sqrt{g} \partial_{y} \phi_{k}=e^{-4 y} \partial_{y} e^{4 y} \partial_{y} \phi_{k}=\left(m^{2}-\lambda_{k}\right) \phi_{k},
$$

using $\sqrt{g} \rightarrow e^{4 y}$. This implies that the leading asymptotic behavior of $\phi_{k}$ is

$$
\phi_{k} \rightarrow e^{\left(-2+\sqrt{4+m^{2}-\lambda_{k}}\right) y} .
$$

On the other hand, if $\phi_{k}$ is normalizable we need

$$
\phi_{k} \leq e^{-2 y}
$$

at infinity. It is possible to satisfy (2.42) with negative $\lambda_{k}$ only if $m^{2}<-4$. 6 The spectrum of eleven-dimensional supergravity on $S^{7}$ has been studied in [17] and has been shown to contain three families of scalars with masses $m^{2}=\frac{1}{4}\left((k-3)^{2}-9\right) ; \frac{1}{4}\left((k+8)^{2}-9\right) ; \frac{1}{4}((k+$ $\left.3)^{2}-9\right)$; and two families of pseudoscalars with masses $m^{2}=\frac{1}{4}\left(k^{2}-9\right) ; \frac{1}{4}\left((k+6)^{2}-9\right)$; where $k=1,2, .$. etc. (Here we have shifted the mass and performed the overall normalization so that conventions of [17] agree with those in [14]). The most negative mass ${ }^{2}$ is $m^{2}=-\frac{9}{4}$, which is insufficient to produce an instability.

6 We assumed here that, as is generically expected, the large $y$ behavior is governed by the dominant exponent 2.41); in principle the coefficient of this term could vanish. 
The theory also contains vector fields with linearized equations of motion

$$
d * d A=0
$$

Consider an ansatz

$$
A=a(y) \sigma_{1} .
$$

Then (2.43) becomes

$$
\left(\partial_{y}^{2} a+2 \partial_{y} a\right) e^{2 y} d y \sigma_{2} \sigma_{3}+\mathcal{O}\left(e^{-2 y}\right) d y \sigma_{2} \sigma_{3}=0 .
$$

This implies that the leading behavior of $A$ is a constant, and that there is no normalizable zero mode of the form (2.44). Similar conclusions apply to $A \sim \sigma_{2}$, while for $A=a \sigma_{3}$ one finds $a \sim e^{y}$, which is also non-normalizable. Hence there are no normalizable zero modes of this form. Allowing for angular dependence of $a$, a negative eigenvalue for (2.43), or considering massive vectors in the theory (which all have $m^{2}>0$ ) only makes it more difficult to get a normalizable eigenmode. In conclusion, the vector fields on $S U(2,1) / U(2)$ also do not induce an instability. We have also checked that normalizable graviton zero modes do not exist, in harmony with the uniqueness theorem [13] discussed in section 2.2. We conclude that $S U(2,1) / U(2) \times S^{7}$ is a stable solution of M-theory.

\subsection{The Boundary Theory}

In accord with the holographic principle, we wish to represent the bulk M-theory on $S U(2,1) / U(2) \times S^{7}$ as a conformal field theory on the conformal boundary of $S U(2,1) / U(2) \times S^{7}$. In this subsection we describe (for scalars) how the operators and correlation functions of this boundary theory can be defined as limits of various bulk quantities. This procedure is a modification of that used to define the boundary theory for $A d S_{4} \times S^{7}$. The resulting correlators are well-behaved and transform appropriately under the boundary conformal group $S U(2,1)$, despite the degeneracy of the boundary metric. In section 4 we discuss possible dual representations in terms of branes.

Let us consider the conformal field theory on the boundary of $S U(2,1) / U(2) \times S^{7}$ with the degenerate metric

$$
d s^{2}=\sigma_{3}^{2}=(d \psi+\cos \theta d \varphi)^{2},
$$

and measure

$$
d^{3} \Omega=\sin \theta d \theta d \psi d \phi .
$$


A conformal transformation is a diffeomorphism together with a Weyl transformation. A field of conformal dimension $\Delta$ transforms as

$$
\delta_{\xi} \mathcal{O}=\left(\mathcal{L}_{\xi}+\frac{\Delta}{3} \nabla_{m} \xi^{m}\right) \mathcal{O}
$$

where $\mathcal{L}_{\xi}$ is the usual Lie derivative, equal to $\xi^{m} \partial_{m}$ acting on scalars. The metric and measure both have $\Delta=-3$.

For simplicity let us restrict our attention to scalar operators $\mathcal{O}$ in the boundary. Let $\delta_{i}$, with $i=1,2, \ldots, 8$, denote the eight $S U(2,1)$ conformal transformations generated by the vectors (2.17)-(2.22) on $S^{3}$. The quadratic Casimir associated to such an operator follows from squaring (2.48) as

$$
C_{I I} \mathcal{O}=g^{i j} \delta_{i} \delta_{j} \mathcal{O}=\frac{4}{9} \Delta(\Delta-3) \mathcal{O}
$$

where $g_{i j}$ is the flat signature $(4,4)$ metric for the $s u(2,1)$ Lie algebra appearing in $(2.32)$. Comparing (2.49) to (2.33) we see that for every scalar field of mass $m$ there is a boundary operator with weight $\Delta$ obeying

$$
m^{2}=\frac{16}{9} \Delta(\Delta-3)
$$

The two-point function of the scalar fields $\left\langle\mathcal{O}_{\Delta_{1}}(z) \mathcal{O}_{\Delta_{2}}(w)\right\rangle$ is fixed by the requirement of invariance under conformal transformations. The requirement of invariance under the isometries generated by $(2.17)-(2.18)$ leads to the following equations:

$$
\begin{aligned}
{\left[h_{1}^{(z)}+h_{1}^{(w)}\right]\left\langle\mathcal{O}_{\Delta_{1}}(z) \mathcal{O}_{\Delta_{2}}(w)\right\rangle } & =0 \\
{\left[h_{2}^{(z)}+h_{2}^{(w)}\right]\left\langle\mathcal{O}_{\Delta_{1}}(z) \mathcal{O}_{\Delta_{2}}(w)\right\rangle } & =0 \\
{\left[l_{1}^{(z)}+l_{1}^{(w)}\right]\left\langle\mathcal{O}_{\Delta_{1}}(z) \mathcal{O}_{\Delta_{2}}(w)\right\rangle } & =0 \\
{\left[\bar{l}_{1}^{(z)}+\bar{l}_{1}^{(w)}\right]\left\langle\mathcal{O}_{\Delta_{1}}(z) \mathcal{O}_{\Delta_{2}}(w)\right\rangle } & =0
\end{aligned}
$$

where the superscripts $(z)$ and $(w)$ on the generators denote the coordinates on $S^{3}$. In order to fully exploit the symmetries, the $S^{3}$ coordinate $z$ is traded for an $S U(2)$ group element $g$ defined by

$$
g_{z}=\left(\begin{array}{cc}
z_{1} & \bar{z}_{2} \\
-z_{2} & \bar{z}_{1}
\end{array}\right)=\left(\begin{array}{cc}
\cos \frac{\theta}{2} e^{i(\varphi+\psi) / 2} & \sin \frac{\theta}{2} e^{i(\varphi-\psi) / 2} \\
-\sin \frac{\theta}{2} e^{-i(\varphi-\psi) / 2} & \cos \frac{\theta}{2} e^{-i(\varphi+\psi) / 2}
\end{array}\right)
$$


Invariance under the $S U(2) \times U(1)$ isometries generated by (2.51) then requires that the correlators are invariant under a left $S U(2)$ action and a right $U(1)$ action on $g$. This requires that the correlator depends only on two real functions or one complex function $U$

$$
\left\langle\mathcal{O}_{\Delta_{1}}(z) \mathcal{O}_{\Delta_{2}}(w)\right\rangle=f(U, \bar{U})
$$

where

$$
\begin{aligned}
U= & \frac{1}{2} \operatorname{Tr}\left[\left(1+\sigma_{3}\right) g_{z}^{\dagger} g_{w}\right] \\
= & \bar{z}_{1} w_{1}+\bar{z}_{2} w_{2} \\
= & \cos \frac{\theta_{z}}{2} \cos \frac{\theta_{w}}{2} \exp \frac{i\left(\varphi_{w}+\psi_{w}-\varphi_{z}-\psi_{z}\right)}{2} \\
& \quad+\sin \frac{\theta_{z}}{2} \sin \frac{\theta_{w}}{2} \exp \frac{-i\left(\varphi_{w}-\psi_{w}-\varphi_{z}+\psi_{z}\right)}{2}
\end{aligned}
$$

and $\bar{U}$ is the conjugate. The requirement for the two-point function to be covariant under the transformation generated by $l_{2}$ is

$$
\left[l_{2}^{(z)}+l_{2}^{(w)}\right]\left\langle\mathcal{O}_{\Delta_{1}}(z) \mathcal{O}_{\Delta_{2}}(w)\right\rangle=-\frac{1}{3}\left[\Delta_{1} \nabla \cdot l_{2}^{(z)}+\Delta_{2} \nabla \cdot l_{2}^{(w)}\right]\left\langle\mathcal{O}_{\Delta_{1}}(z) \mathcal{O}_{\Delta_{2}}(w)\right\rangle,
$$

which can be rewritten in the form

$$
\bar{z}_{1}\left(-\frac{2}{3} \Delta f+(1-U) \partial_{U} f\right)+\bar{w}_{1}\left(-\frac{2}{3} \Delta f+(1-\bar{U}) \partial_{\bar{U}} f\right)=0,
$$

where $\Delta=\Delta_{1}=\Delta_{2}$. The condition that $\Delta_{1}=\Delta_{2}$ follows from comparing equation (2.55) with its conjugate. Note that $\nabla \cdot l_{2} \equiv \frac{1}{\sqrt{g}} \partial_{i}\left(\sqrt{g} l_{2}^{i}\right)=2 \bar{z}_{1}$, where $\sqrt{g} \sim \sin \theta$.

The other three equations have $\left(z_{1}, w_{1}\right),\left(\bar{z}_{2}, \bar{w}_{2}\right)$ and $\left(z_{2}, w_{2}\right)$ consecutively, in place of $\left(\bar{z}_{1}, \bar{w}_{1}\right)$. The function

$$
f(U, \bar{U})=|1-U|^{-\frac{4 \Delta}{3}}
$$

satisfies (2.56). Since moreover each term in front of $\bar{z}_{1}$ and $\bar{w}_{1}$ vanishes separately, this function satisfies evidently all the other equations. Thus we have found that the two-point function of two scalar fields of dimension $\Delta$ is given by

$$
\left\langle\mathcal{O}_{\Delta}(z) \mathcal{O}_{\Delta}(w)\right\rangle=\frac{\text { const }}{|1-U|^{\frac{4 \Delta}{3}}} .
$$

In the preceding we saw that conformal invariance determines the two-point functions of the boundary operators. Higher-point functions will not be fully determined by conformal invariance. The recipe for calculating a general correlation function within the 
$A d S / C F T$ correspondence, as formulated in [14,15], is the following: first, compute the supergravity partition function in terms of the boundary values of the fields; then, identify the operators in the boundary conformal field theory whose sources are the given boundary values; finally, interpret the supergravity partition function as a generating functional of those operators. This prescription associates to each field $\phi$ in the supergravity action a corresponding operator $\mathcal{O}$ by the relation

$$
\left\langle e^{\int_{\partial} \phi_{0} \mathcal{O}}\right\rangle=e^{-I(\phi)} .
$$

Here $I(\phi)$ is the classical action evaluated on the solutions of the supergravity equation of motion subject to some boundary condition, the integral is over the boundary, and the left hand side is interpreted as a partition function of the connected Green functions for the operators $\mathcal{O}$. Because of the boundary degeneracy, it is not manifestly obvious that this prescription can be adapted to $S U(2,1) / U(2)$. In this subsection we see that the divergences cancel and the prescription can indeed be adapted.

Let us consider a scalar field of mass $m$ in the bulk of $S U(2,1) / U(2)$. We will not keep track of finite normalization constants in the rest of this section. In order to compute the correlation function of the operators $\mathcal{O}$, we first have to calculate the action

$$
I(\phi)=\int d r d \theta d \psi d \varphi \sqrt{g}\left((\nabla \phi)^{2}+m^{2} \phi^{2}\right)
$$

for a solution of a classical equation of motion

$$
\nabla^{2} \phi=m^{2} \phi
$$

subject to the boundary condition

$$
\lim _{r \rightarrow 1} \phi(r, \theta, \psi, \varphi)=\left(1-r^{2}\right)^{2-\frac{2 \Delta}{3}} \phi_{0}(\theta, \psi, \varphi) .
$$

We use the metric (2.4) in the bulk of $S U(2,1) / U(2)$ and the relation between the mass $m$ of the scalar field and the dimension $\Delta$ of the boundary operator, $m^{2}=\frac{16}{9} \Delta(\Delta-3)$. The solution of (2.61) is given by

$$
\phi(r, \theta, \psi, \varphi)=\int K\left(r, \theta, \psi, \varphi ; \theta^{\prime}, \psi^{\prime}, \varphi^{\prime}\right) \phi_{0}\left(\theta^{\prime}, \psi^{\prime}, \varphi^{\prime}\right) \sin \theta^{\prime} d \theta^{\prime} d \psi^{\prime} d \varphi^{\prime}
$$

where the bulk-to-boundary propagator is

$$
K\left(r, \theta, \psi, \varphi ; \theta^{\prime}, \psi^{\prime}, \varphi^{\prime}\right)=\frac{\left(1-r^{2}\right)^{\frac{2 \Delta}{3}}}{|1-r U|^{\frac{4 \Delta}{3}}}
$$


with $U=\cos \frac{\theta}{2} \cos \frac{\theta^{\prime}}{2} \exp \frac{i\left(\varphi^{\prime}+\psi^{\prime}-\varphi-\psi\right)}{2}+\sin \frac{\theta}{2} \sin \frac{\theta^{\prime}}{2} \exp \frac{-i\left(\varphi^{\prime}-\psi^{\prime}-\varphi+\psi\right)}{2}$, as above. Note that

$$
\lim _{r \rightarrow 1} K\left(r, \theta, \psi, \varphi ; \theta^{\prime}, \psi^{\prime}, \varphi^{\prime}\right)=\frac{\left(1-r^{2}\right)^{2-\frac{2 \Delta}{3}}}{\sin \theta^{\prime}} \delta\left(\theta^{\prime}-\theta\right) \delta\left(\psi^{\prime}-\psi\right) \delta\left(\varphi^{\prime}-\varphi\right) .
$$

Upon integrating by parts, we find that only the boundary term contributes to the action $(2.60)$ :

$$
\begin{gathered}
I(\phi)=\lim _{r \rightarrow 1} \int d^{3} \Omega \frac{1}{\left(1-r^{2}\right)} \phi \partial_{r} \phi . \\
I(\phi)=-\int d^{3} \Omega d^{3} \Omega^{\prime} \frac{\phi_{0}(\theta, \varphi, \psi) \phi_{0}\left(\theta^{\prime}, \varphi^{\prime}, \psi^{\prime}\right)}{|1-U|^{\frac{4 \Delta}{3}}} .
\end{gathered}
$$

We see that the boundary action is indeed a finite function of $\phi_{0}$, despite the degeneracy of the boundary metric, and that it correctly reproduces the two-point function of $\mathcal{O}$ as determined by conformal invariance in the preceding subsection. In principle this boundary action can also be used to determine the higher-point correlation functions of $\mathcal{O}$ and might also be extended to fields of higher spin.

\section{Supersymmetric Lorentzian Cosets}

In this section we consider compactifications of IIB on $S O(2,2) / S O(2) \times S^{5}$ and Mtheory on $S O(3,2) / S O(3) \times S^{4}$, where $S O(2,2) / S O(2) \equiv W_{4,2}$ and $S O(3,2) / S O(3) \equiv$ $W_{5,2}$ are the noncompact cousins of the Stiefel manifolds $S O(4) / S O(2) \equiv V_{4,2}$ and $S O(5) / S O(3) \equiv V_{5,2}$. Each of these spaces is defined with the divisor subgroup embedded canonically in the larger group. These examples differ from that of the previous section in that they have Lorentzian signature and are supersymmetric. The unbroken supersymmetries are described in subsection 3.1. The geometry of $W_{4,2}$ and its conformal boundary are detailed in subsection 3.2 . In 3.3 scalar fields in $W_{4,2}$ are described.

\subsection{Supersymmetry}

In this subsection it is shown that the spaces $W_{5,2} \times S^{7}$ and $W_{4,2} \times S^{5}$ preserve the same amount of supersymmetry as $A d S_{4} \times V_{5,2}$ and $A d S_{5} \times V_{4,2}$, respectively, namely eight supersymmetries in all cases.

On a space with nonvanishing cosmological constant, unbroken supersymmetries are constructed from solutions of the Killing spinor equation

$$
D_{m} \eta=0
$$


where $D_{m}=\nabla_{m}-i \Gamma_{m}$. The integrability condition for this equation is that the operator $\left[D_{m}, D_{n}\right]=\frac{1}{4} C_{m n}{ }^{a b} \Gamma_{a b}$ has zero modes, where $C_{m n}{ }^{a b}$ is the Weyl tensor. Hence we are interested in the holonomy of the Weyl tensor.

We first recall that the Weyl holonomy of $V_{5,2}$ is $S U(3)$. Following the conventions and methodology of [18], define $\left(T^{A B}\right)_{C D}=\delta_{C}^{A} \delta_{D}^{B}-\delta_{D}^{A} \delta_{C}^{B}$ to be the generators of $S O(5)$, where $A, B, C, D$ range from 1 to 5 . To make the canonical embedding of $S O(3)(\overrightarrow{5} \rightarrow \overrightarrow{3}+\overrightarrow{1}+\overrightarrow{1})$ manifest, rewrite the generators as

$$
X^{i}=\frac{1}{2} \epsilon^{i j k} T^{j k}, X^{m}=T^{4 m}, X^{\hat{m}}=T^{5 m}, X^{0}=T^{45},
$$

where $X^{i}$ generate the $S O(3)$ subgroup, and the indices $i, m, \hat{m}$ range from 1 to 3 . The values of the nonvanishing structure constants of $S O(5)$ (defined by $\left[T_{A}, T_{B}\right]=C_{A B}^{C} T_{C}$ ) are then

$$
\begin{gathered}
C_{i j}^{k}=\epsilon_{i j k}, \quad C_{i m}^{n}=C_{i \hat{m}}^{\hat{n}}=-\epsilon_{i m n}, \quad C_{m 0}^{\hat{n}}=-C_{\hat{m} 0}^{n}=\delta_{m}^{n}, \\
C_{m n}^{i}=C_{\hat{m} \hat{n}}^{i}=-\epsilon_{i m n}, \quad C_{m \hat{n}}^{0}=-\delta_{n}^{m} .
\end{gathered}
$$

The metric on $G / H$ inherited from the group-invariant metric on $G$ is not in general an Einstein metric, but can sometimes be transformed into an Einstein metric without losing any isometries by appropriately rescaling the vielbein components. Consider the matrices of structure constants $\left(C_{D}\right)_{b}{ }^{a}$, as $D$ runs over the indices in the normalizer of the subgroup, and $a$ and $b$ run over flat coset indices. It was shown in 18 that if these matrices are block diagonal in the spaces spanned by the vielbein components $e^{a_{1}}, e^{a_{2}}, \ldots$, then an arbitrary rescaling of the vielbein, $e^{a_{i}} \rightarrow r\left(a_{i}\right) e^{a_{i}}$, preserves the original isometries. One can try to find a rescaling to obtain an Einstein metric on the coset space. For $V_{5,2}$, rescale with $r(m)=r(\hat{m})=4$ and $r(0)=\sqrt{\frac{32}{3}}$.

The Riemann tensor for the rescaled coset can be calculated using the Maurer-Cartan equations and the Jacobi identities for the products of structure constants. In terms of the structure constants and squashing parameters, the Riemann tensor is

$$
\begin{array}{r}
R_{b d e}^{a}=\frac{1}{4} C_{b c}^{a} C_{d e}^{c}\left(\begin{array}{c}
a b \\
c
\end{array}\right) \frac{r(d) r(e)}{r(c)}+\frac{1}{2} C_{b i}^{a} C_{d e}^{i} r(d) r(e)+ \\
\frac{1}{8} C_{c d}^{a} C_{b e}^{c}\left(\begin{array}{c}
a c \\
d
\end{array}\right)\left(\begin{array}{cc}
b c \\
e
\end{array}\right)-\frac{1}{8} C_{c e}^{a} C_{b d}^{c}\left(\begin{array}{cc}
a & c \\
e
\end{array}\right)\left(\begin{array}{cc}
b c \\
d
\end{array}\right),
\end{array}
$$

with

$$
\left(\begin{array}{c}
a b \\
c
\end{array}\right) \equiv \frac{r(a) r(c)}{r(b)}+\frac{r(b) r(c)}{r(a)}-\frac{r(a) r(b)}{r(c)}
$$


where $a, b, \ldots$ are the flat $G / H$ coset indices, namely $m, \hat{m}, 0$, and $i$ is an $H$ index. The flat metric is defined as $\gamma_{a b}=-C_{a D}^{C} C_{b C}^{D}$. The nonvanishing components of the Weyl tensor for $V_{5,2}$ read

$$
C_{p q}^{m n}=C_{\hat{p} \hat{q}}^{\hat{m} \hat{n}}=C_{\hat{p} \hat{q}}^{m n}=5\left(\delta_{p}^{m} \delta_{q}^{n}-\delta_{q}^{m} \delta_{p}^{n}\right), \quad C_{p \hat{q}}^{m \hat{n}}=2 \delta_{n}^{m} \delta_{q}^{p}-3 \delta_{q}^{m} \delta_{p}^{n}-3 \delta_{p}^{m} \delta_{q}^{n} .
$$

The holonomy of $V_{5,2}$ is $S U(3)$ if there exists a two-form $J$ such that

$$
C_{A B}{ }_{N}^{M} J_{P}^{N}=C_{A B}{ }_{P} J^{M}{ }_{N}, \quad C_{A B}{ }_{N}^{M} J_{M}{ }^{N}=0
$$

The form with the components

$$
J_{m}^{n}=J_{\hat{m}}^{\hat{n}}=0, \quad J_{m}^{\hat{n}}=-J_{\hat{n}}^{m}=\delta_{n}^{m}
$$

satisfies equations (3.8) with the Weyl tensor of (3.7). Thus, the holonomy of $V_{5,2}$ is $S U(3)$. The spinor $\overrightarrow{8}$ of $\operatorname{spin}(7)$ decomposes as $\overrightarrow{8}=\overrightarrow{1}+\overrightarrow{1}+\overrightarrow{3}+\overrightarrow{3}^{*}$ under $S U(3)$. The two singlets account for two covariantly constant spinors on $V_{5,2}$. The full symmetry group for M-theory on $A d S_{4} \times V_{5,2}$ is $O S p(2,2 \mid 2) \times S O(5)$, which has 8 supercharges.

Similar arguments are valid for $V_{4,2}$, obtained by the canonical embedding of $S O(2)$ in $S O(4): \overrightarrow{4} \rightarrow \overrightarrow{2}+\overrightarrow{1}+\overrightarrow{1}$. Upon calculating the Weyl tensor, one finds that the holonomy of $V_{4,2}$ is $S U(2)$. This eliminates half the supersymmetries, but, due to chirality constraints, these can be used to construct only 8 supercharges for IIB string theory on $A d S_{5} \times V_{4,2}$ [19]. The full symmetry group is $S U(2,2 \mid 1) \times S O(4)$.

Now consider $W_{4,2}$ and $W_{5,2}$. To obtain the generators of $S O(n-2,2)$ from $S O(n)$, simply multiply the generators $X^{m}$ and $X^{\hat{m}}$ by $i$, so that only the structure constants in (3.4) will change sign while those in (3.3) remain the same. Note also that only the $\gamma_{00}$ component of the flat metric changes sign. From (3.5), we find that $R_{c d}^{a b}\left(W_{n, 2}\right)=$ $-R^{a b}{ }_{c d}\left(V_{n, 2}\right)$. Therefore, $C^{a b}{ }_{c d}\left(W_{n, 2}\right)=-C^{a b}{ }_{c d}\left(V_{n, 2}\right)$. Since the " 0 " components of the Weyl tensor all vanish according to (3.7), the flat metric on the algebra generated by the Weyl tensors of $W_{n, 2}$ and $V_{n, 2}$ are the same. Hence the holonomies of $W_{4,2}$ and $W_{5,2}$ are $S U(2)$ and $S U(3)$ respectively. The full symmetry groups of compactifications IIB $\left.\right|_{W_{4,2} \times S^{5}}$ and $\left.\mathrm{M}\right|_{W_{5,2} \times S^{4}}$ are $S U(4 \mid 1) \times S O(2,2)$ and $O S p(4 \mid 2) \times S O(3,2)$ respectively, both of which have 8 supercharges. 


\subsection{Geometry of $W_{4,2} \equiv S O(2,2) / S O(2)$ and its boundary}

The coset space obtained by quotienting $S O(2,2)$ by the $S O(2)$ subgroup is a symmetric Einstein space with negative cosmological constant and signature $(4,1)$. Topologically $W_{4,2}$ is $S^{1} \times \mathbf{R}^{4}$, so that $\pi_{1}\left(W_{4,2}\right)=\mathbf{Z}$.

The Riemannian metric on $W_{4,2}$ can be obtained by an analytic continuation of the $V_{4,2}$ metric and takes the form

$$
d s^{2}=-\frac{1}{9}\left(d \psi+\cosh y_{1} d \varphi_{1}+\cosh y_{2} d \varphi_{2}\right)^{2}+\frac{1}{6}\left(d y_{1}^{2}+\sinh ^{2} y_{1} d \varphi_{1}^{2}+d y_{2}^{2}+\sinh ^{2} y_{2} d \varphi_{2}^{2}\right)
$$

where $y_{i} \in[0, \infty), \varphi_{i} \in[0,2 \pi)$, and $\psi \in[0,4 \pi)$. The coordinate $\psi$ parametrizes the $U(1)$ fiber of $W_{4,2}$ viewed as a $U(1)$ bundle over $A d S_{2} \times A d S_{2}$. The isometries of this space are generated by seven Killing vectors

$$
\begin{gathered}
L_{0}^{i}=i \partial_{\varphi_{i}}, \quad J=i \partial_{\psi} \\
L_{-1}^{i}=i e^{-i \varphi_{i}}\left(\operatorname{coth} y_{i} \partial_{\varphi_{i}}-\frac{1}{\sinh y_{i}} \partial_{\psi}+i \partial_{y_{i}}\right) \\
L_{1}^{i}=i e^{i \varphi_{i}}\left(\operatorname{coth} y_{i} \partial_{\varphi_{i}}-\frac{1}{\sinh y_{i}} \partial_{\psi}-i \partial_{y_{i}}\right)
\end{gathered}
$$

which generate an $s o(2,2) \times s o(2)$ algebra $(s o(2,2) \cong \operatorname{sl}(2, R) \times \operatorname{sl}(2, R))$ :

$$
\left[L_{0}^{i}, L_{ \pm 1}^{j}\right]=\mp \delta^{i j} L_{ \pm 1}^{i}, \quad\left[L_{1}^{i}, L_{-1}^{j}\right]=2 \delta^{i j} L_{0}^{i}, \quad\left[L_{0}^{i}, J\right]=\left[L_{1}^{i}, J\right]=\left[L_{-1}^{i}, J\right]=0
$$

where $i=1,2$.

The boundary of $W_{4,2}$ might be defined by

$$
\sinh ^{2} y_{1}+\sinh ^{2} y_{2}=\Lambda^{2} \rightarrow \infty
$$

which can be written in terms of a new coordinate $\chi \in\left[0, \frac{\pi}{2}\right)$ as

$$
\sinh y_{1}=\Lambda \cos \chi, \quad \sinh y_{2}=\Lambda \sin \chi
$$

The conformal boundary metric is

$$
d s^{2}=\cos ^{2} \chi d \varphi_{1}^{2}+\sin ^{2} \chi d \varphi_{2}^{2}-4 \cos \chi \sin \chi d \varphi_{1} d \varphi_{2}
$$

The $U(1)$ fiber parametrized by $\psi$ has shrunk to zero size in the conformal metric (along with the $S^{5}$ which we have suppressed). The conformal Killing vectors of the boundary, 
which form an $s o(2,2)$ algebra, are that subset of (3.11) that act nontrivially on the boundary.

We have not succeeded in making sense of the notion of a theory on the boundary (3.15). 0 In most locations it is a degenerate signature $(+,-, 0)$ metric, but at $\chi=0, \frac{\pi}{2}$, it degenerates further to signature $(+, 0,0)$.

An alternate procedure that yields a smoother result is to suppress the $\chi$ coordinate. A motivation for this is that distances in the $\chi$ direction are all zero, together with those along with the $U(1)$ fiber and the $S^{5}$, in the conformal boundary metric. The variable $\chi$ is eliminated in the two-dimensional (rather than three-dimensional) "boundary" defined by

$$
\sinh ^{2} y_{1}=\sinh ^{2} y_{2}=\Lambda^{2} \rightarrow \infty
$$

which is simply $T^{2}$ with the conformal boundary metric

$$
d s^{2}=d u_{1}^{2}-d u_{2}^{2}
$$

where $u_{1}=\varphi_{1}-2 \varphi_{2}$ and $u_{2}=\sqrt{3} \varphi_{2}$. The $s o(2,2)$ algebra on the boundary is generated by the conformal Killing vectors

$$
l_{0}^{i}=i \partial_{u_{i}}, \quad l_{-1}^{i}=i e^{-i u_{i}} \partial_{u_{i}}, \quad l_{1}^{i}=i e^{i u_{i}} \partial_{u_{i}}
$$

This algebra can be extended to two copies of Virasoro algebra with the generators

$$
l_{n}^{i}=i e^{i n u_{i}} \partial_{u_{i}}
$$

Hence we may expect a two-dimensional conformal field theory at the boundary.

A novel feature of this spacetime is the existence of closed timelike curves. Examples are the curves $\chi=\frac{\pi}{4}, \varphi_{1}=\varphi_{2}$, constant $\psi$ and large $y$. Unlike in $A d S_{4}$ these cannot be eliminated by going to the covering space.

\footnotetext{
7 Similar issues arise in other examples such as IIB string theory on $A d S_{2} \times A d S_{3} \times S^{5}$.
} 


\subsection{Scalar Fields in $W_{4,2}$}

In this subsection we derive the relation between the mass $m$ of the scalar field in the bulk and the highest weights $j, h_{1}, h_{2}$ of the $s o(2,2) \times s o(2)$ algebra.

The scalar field $\phi$ in the bulk of $S O(2,2) / S O(2)$ is described by the wave equation

$$
\nabla^{2} \phi=m^{2} \phi
$$

where $\nabla^{2}$ is the Laplacian for the metric (3.10),

$$
\begin{array}{r}
\nabla^{2}=3\left(\frac{2}{\sinh ^{2} y_{1}} \partial_{\varphi_{1} \varphi_{1}}+\frac{2}{\sinh ^{2} y_{2}} \partial_{\varphi_{2} \varphi_{2}}-\frac{4 \operatorname{coth} y_{1}}{\sinh y_{1}} \partial_{\varphi_{1} \psi}-\frac{4 \operatorname{coth} y_{2}}{\sinh y_{2}} \partial_{\varphi_{2} \psi}+3 \partial_{\psi \psi^{-}}-\right. \\
\left.2 \operatorname{coth}^{2} y_{1} \partial_{\psi \psi}-2 \operatorname{coth}^{2} y_{2} \partial_{\psi \psi}+2 \operatorname{coth} y_{1} \partial_{y_{1}}+2 \partial_{y_{1} y_{1}}+2 \operatorname{coth} y_{2} \partial_{y_{2}}+2 \partial_{y_{2} y_{2}}\right) .
\end{array}
$$

The Laplacian can be written in terms of the Casimir of $s o(2,2) \times s o(2)$ as

$$
\nabla^{2}=-6\left(\sum_{i=1}^{2}\left(\frac{1}{2}\left\{L_{1}^{i}, L_{-1}^{i}\right\}-L_{0}^{i 2}\right)+\frac{1}{2} J^{2}\right) .
$$

Highest weight states $|h\rangle$ are characterized by

$$
L_{0}^{i}|h\rangle=h_{i}|h\rangle, \quad J|h\rangle=j|h\rangle, \quad L_{1}^{i}|h\rangle=0
$$

Acting with the Casimir operator (3.22) on the highest weight state $|h\rangle$ leads to the relation

$$
m^{2}=6\left(h_{1}\left(h_{1}-1\right)+h_{2}\left(h_{2}-1\right)-j^{2} / 2\right)
$$

Hence for every scalar field $\phi$ of mass $m$ we expect operators $\mathcal{O}$ in the boundary theory with corresponding weights.

\section{Brane constructions}

The holographic principle suggests that $\mathrm{M} /$ string theory on a given space can be represented as a field theory on the boundary of the space. In the preceding section, following the logic of the $A d S / C F T$ correspondence, the boundary correlators for various cosets have been described as limits of bulk correlators. In some of the $A d S$ cases, dual description of the boundary theory for example as a large $N$ gauge theory, are possible. In this section such dual descriptions will be considered for the cases at hand. 
The field theory on the boundary of $A d S_{4} \times S^{7}$ can be defined as the infrared limit of a theory of M2-branes, or the strong-coupling, infrared limit of the D2-brane gauge theory. This theory lives on $S^{3}$ with the round metric. One may consider the same limit on $S^{3}$ with the squashed metric

$$
d s^{2}=\sigma_{3}^{2}+\frac{1}{a}\left(\sigma_{1}^{2}+\sigma_{2}^{2}\right) .
$$

It is natural to conjecture that in the limit that the squashing parameter $a$ is taken to infinity, one obtains the dual description of M-theory on $S U(2,1) / U(2) \times S^{7}$. (A similar conjecture was advanced in the context of Taub-Nut where a finitely squashed $S^{3}$ is encountered [8,9].) The results of section 2 can be regarded as evidence that this limit is well-defined. ${ }^{2}$ Similar conjectures for $W_{4,2}$ and $W_{5,2}$ involve Yang-Mills theory on a degenerate four-geometry and the $(0,2)$ fivebrane conformal field theory on a degenerate six-geometry. While perhaps plausible, these descriptions do not seem terribly useful in their present formulation and are therefore unsatisfying.

It would be illuminating to find these or other noncompact coset spaces as nearhorizon geometries of brane configurations. The branes may have nontrivial worldvolume geometry and/or internal field excitations. The spacetime supergravity solution for such brane configurations is not in general known. However it may be possible in some cases with enough symmetry to find the near horizon geometry without knowing the full spacetime solution. One construction that may lead to noncompact coset spaces - although perhaps not the ones explicitly discussed in this paper-involves the spontaneous breakdown of conformal invariance. This can occur in the presence of solitons. The generators of the conformal group $S O(D, 2)$ of $D$-dimensional Minkowski space are

$$
v^{a}=\lambda x^{a}+b^{a} x^{2}-2 x^{a} b \cdot x
$$

together with the Poincare generators. A scalar field $\phi$ for example transforms as

$$
\delta \phi=v^{a} \partial_{a} \phi+\frac{D-2}{2 D} \partial_{a} v^{a} \phi
$$

8 Free field theory partition functions on this space are computed in 20].

9 The scalar curvature of the metric (4.1) is $R=2-\frac{1}{2 a}$, and so is negative for the $S U(2,1) / U(2)$ (as well as Taub-Nut) boundary metric. This will lead to Coulomb-branch instabilities near the origin for the gauge theory scalars due to the $R \phi^{2}$ coupling. Hence the flow into the infrared could be quite nontrivial, and there may be subtleties concerning the order in which the infrared and $a \rightarrow 0$ limit are taken. 
A given expectation for $\phi$ breaks the conformal group down to a subgroup generated by those $v$ 's that annihilate $\phi$ in (4.3). Unbroken global scale invariance (generated by $v=\lambda x$ ) requires

$$
x^{a} \partial_{a} \phi=-\frac{D-2}{2} \phi,
$$

so $\phi$ must scale in the specified way with $x$. In general this implies for $D>2$ that $\phi$ will be singular at the origin. Now consider the special conformal transformations parametrized by the vector $b^{a}$ in (4.2). If $\phi$ is invariant under some translations so that for longitudinal transformations $b_{L}^{a} \partial_{a} \phi=0$, then (4.4) is necessary and sufficient to ensure invariance under the associated special conformal transformations. The transverse transformations with $b_{T}^{a} \partial_{a} \phi \neq 0$ are necessarily broken.

In summary, if the field configuration $\phi$ scales as (4.4) and is invariant under $d$ dimensional Poincare transformations, it follows that the conformal group $S O(D, 2)$ is broken down to $S O(d, 2)$. An obvious generalization of this statement pertains to the brane worldvolume metric as well as higher-rank tensor fields. Further conditions should be imposed if supersymmetry is to be preserved.

In general, there are many noncompact cosets of which only three examples were discussed in this paper. One obvious generalization is to quotient by both a left and a right action. There are also many ways to spontaneously break conformal invariance with solitons or nontrivial induced metrics on a brane worldvolume. It would be interesting to find a plausible candidate for a dual pair.

\section{Acknowledgements}

We are grateful to M. Duff, S. Gubser, G. Horowitz, R. Kallosh, J. Maldacena, J. Polchinski, C. Pope, M. Spradlin, E. Witten and S.-T. Yau for useful conversations. It has come to our attention that related ideas have been independently pursued by A. Mikhailov and also by M. Taylor-Robinson. This work was supported in part by DOE grant DEFGO2-91ER40654 and an NDSEG graduate fellowship.

\section{Appendix A. $S U(2,1)$ commutation relations}

The relations between the generators (2.7)-(2.10) and the standard ones $F_{i}$ are

$$
\begin{gathered}
F_{1}=\frac{1}{2}\left(L_{1}-\bar{L}_{1}\right), \quad F_{2}=-\frac{i}{2}\left(L_{1}+\bar{L}_{1}\right), \quad F_{3}=\frac{1}{2}\left(H_{2}-H_{1}\right), \quad F_{8}=-\frac{\sqrt{3}}{2}\left(H_{1}+H_{2}\right), \\
F_{4}=-\frac{1}{2}\left(L_{2}+\bar{L}_{2}\right), \quad F_{5}=\frac{i}{2}\left(L_{2}-\bar{L}_{2}\right), \quad F_{6}=\frac{1}{2}\left(L_{3}+\bar{L}_{3}\right), \quad F_{7}=-\frac{i}{2}\left(L_{3}-\bar{L}_{3}\right) .
\end{gathered}
$$


The standard generators $F_{i}$ satisfy

$$
\left[F_{i}, F_{j}\right]=i f_{i j k} F_{k}
$$

with $f_{123}=1, f_{147}=1 / 2, f_{156}=-1 / 2, f_{246}=1 / 2, f_{257}=1 / 2, f_{345}=1 / 2, f_{367}=-1 / 2$, $f_{458}=\sqrt{3} / 2, f_{678}=\sqrt{3} / 2$.

\section{Appendix B. Conformal Killing vectors for the $S U(2,1) / U(2)$ boundary}

The conformal Killing vectors of the boundary $\xi^{k}$ should satisfy the following equation

$$
\mathcal{L}_{\xi} \sigma_{3}=\hat{f}(\theta, \varphi, \psi) \sigma_{3}
$$

where $\mathcal{L}$ is the Lie derivative along the vector field $\xi$. In components, (B.1) takes the form

$$
\begin{gathered}
\partial_{\theta} \xi^{\psi}+\cos \theta \partial_{\theta} \xi^{\varphi}=0 \\
-\sin \theta \xi^{\theta}+\partial_{\varphi} \xi^{\psi}+\cos \theta \partial_{\varphi} \xi^{\varphi}=\hat{f} \cos \theta \\
\partial_{\psi} \xi^{\psi}+\cos \theta \partial_{\psi} \xi^{\varphi}=\hat{f} .
\end{gathered}
$$

The solution of the above equations with $\hat{f}=f e^{\alpha \varphi+\beta \psi} \neq 0$ is given by the following set of vectors, parametrized by two numbers $\alpha, \beta$ and a function $f(\theta)$,

$$
L_{f}^{\alpha \beta}=\frac{e^{\alpha \varphi+\beta \psi}}{\beta \sin \theta}\left[f(\theta)(\alpha-\beta \cos \theta) \partial_{\theta}-f_{\theta}(\theta) \partial_{\varphi}+\left(f(\theta) \sin \theta+f_{\theta}(\theta) \cos \theta\right) \partial_{\psi}\right]
$$

For $\hat{f}=0$ we get

$$
H_{g}^{a}=e^{a \varphi}\left[-\frac{a}{\sin \theta}\left\{\int d \theta g(\theta) \sin \theta+C\right\} \partial_{\theta}+g(\theta) \partial_{\varphi}-\left\{\int d \theta \cos \theta g_{\theta}(\theta)+C\right\} \partial_{\psi} \cdot\right]
$$

Here $\alpha, \beta, a$ and $C$ are arbitrary constants and $f(\theta), g(\theta)$ are arbitrary functions of $\theta$ such that the corresponding Killing vectors are nonsingular. These generators enlarge the $S U(2,1)$ algebra of conformal Killing vectors. 


\section{References}

[1] J. Maldacena, "The Large $N$ Limit of Superconformal Field Theories and Supergravity," Adv. Theor. Math. Phys. 2 (1998) 231, hep-th/9711200.

[2] J. D. Bekenstein, "Black Holes and the Second Law," Nuovo Cim. Lett. 4 (1972) 737.

[3] G. 't Hooft, "Dimensional Reduction in Quantum Gravity," in Salaamfest 1993, p. 284, gr-qc/9310026.

[4] L. Susskind, "The World as a Hologram," J. Math. Phys. 36 (1995) 6377, hepth/9409089.

[5] W. Fischler and L. Susskind, "Holography and Cosmology," hep-th/9806039; N. Kaloper and A. Linde, "Cosmology vs. Holography," hep-th/9904120; R. Easther and D. A. Lowe, "Holography, Cosmology and the Second Law of Thermodynamics," hepth/9902088.

[6] T. Banks, W. Fischler, S. Shenker and L. Susskind, "M-theory as a matrix model: a conjecture," Phys. Rev. D55 (1997) 5112, hep-th/9610043.

[7] E. Witten, "Anti-de Sitter Space, Thermal Phase Transition, and Confinement In Gauge Theories," Adv. Theor. Math. Phys. 2 (1998) 505, hep-th/9803131.

[8] A. Chamblin, R. Emparan, C. Johnson and R. Myers, "Large N Phases, Gravitational Instantons and the Nuts and Bolts of AdS Holography," Phys. Rev. D59 (1999) 64010, hep-th/9808177.

[9] S. W. Hawking, C. J. Hunter and D. N. Page, "Nut Charge, Anti-de Sitter Space and Entropy," Phys. Rev. D59 (1999) 44033, hep-th/9809035.

[10] S. W. Hawking, C. J. Hunter and M. M. Taylor-Robinson, "Rotation and the AdS/CFT correspondence," Phys. Rev. D59 (1999) 064005, hep-th/9811056.

[11] I. Klebanov and E. Witten, "Superconformal Field Theory on Threebranes at a Calabi-Yau Singularity," Nucl. Phys. B536 (1998) 199, hep-th/9807080; S. Gubser, "Einstein manifolds and Conformal Field Theory," Phys. Rev. D59 (1999) 25006 , hep-th/9807164; M. J. Duff, H. Lu and C. N. Pope, " $A d S_{3} \times S^{3}$ (Un)twisted and Squashed, and an $O(2,2 ; Z)$ Multiplet of Dyonic Strings," Nucl. Phys. B544 (1999) 145, hep-th/9807173; B. S. Acharya, J. M. Figueroa-O'Farrill, C. M. Hull and S. Spence, "Branes at conical singularities and holography," Adv. Theor. Math. Phys. 2 (1998) 1249, hep-th/9808014; D. R. Morrison and M. R. Plesser, "Non-Spherical Horizons, I," hep-th/9810201.

[12] C. L. Fefferman, "Monge-Ampère equations, the Bergman kernel, and geometry of pseudoconvex domains," Ann. of Math. 103 (1976) 395.

[13] S. Y. Cheng and S.-T. Yau, "On the Existence of a Complete Kähler Metric on NonCompact Complex Manifolds and the Regularity of Fefferman's Equation," Comm. Pure Appl. Math. 33 (1980) 507. 
[14] E. Witten, "Anti-de Sitter space and holography," Adv. Theor. Math. Phys. 2 (1998) 253, hep-th/9802150.

[15] S. Gubser, I. Klebanov and A. Polyakov, "Gauge theory correlators from noncritical string theory," Phys. Lett. B428 (1998) 105, hep-th/9802109.

[16] I. Bars and Z. Teng, "The unitary irreducible representations of $S U(2,1)$, , J. Math. Phys. 31 (1990) 7.

[17] B. Biran, A. Casher, F. Englert, M. Rooman and P. Spindel, "The Fluctuating Seven Sphere in Eleven-Dimensional Supergravity," Phys. Lett. 134B (1984) 179.

[18] L. Castellani, L. J. Romans and N. P. Warner, "Symmetries of Coset Spaces and Kaluza-Klein Supergravity, “ Ann. Phys. 157 (1984) 394; L. Castellani, L. J. Romans and N. P. Warner, "A Classification of Compactifying Solutions for d=11 Supergravity," Nucl. Phys. B241 (1984) 429.

[19] L. Romans, "New Compactifications of Chiral N=2, d=10 Supergravity," Phys. Lett. 153B (1985) 392.

[20] J. S. Dowker, "Effective actions on the squashed three-sphere," hep-th/9812202. 